\title{
A Genetically Mediated Bias in Decision Making Driven by Failure of Amygdala Control
}

\author{
Jonathan P. Roiser, ${ }^{1}$ Benedetto de Martino, ${ }^{2}$ Geoffrey C. Y. Tan, ${ }^{2,3}$ Dharshan Kumaran, ${ }^{2}$ Ben Seymour, ${ }^{2,4}$ \\ Nicholas W. Wood, ${ }^{3}$ and Raymond J. Dolan ${ }^{2}$ \\ ${ }^{1}$ Institute of Cognitive Neuroscience, University College London, London WC1N 3AR, United Kingdom, ${ }^{2}$ Wellcome Trust Centre for Neuroimaging and \\ ${ }^{3}$ Department of Molecular Neuroscience, Institute of Neurology, University College London, London WC1N 3BG, United Kingdom, and ${ }^{4}$ Economic and \\ Social Research Council Centre for Economic Learning and Social Evolution, Department of Economics, University College London, London WC1E 6BT, \\ United Kingdom
}

Genetic variation at the serotonin transporter-linked polymorphic region (5-HTTLPR) is associated with altered amygdala reactivity and lack of prefrontal regulatory control. Similar regions mediate decision-making biases driven by contextual cues and ambiguity, for example the "framing effect." We hypothesized that individuals hemozygous for the short (s) allele at the 5-HTTLPR would be more susceptible to framing. Participants, selected as homozygous for either the long (la) or s allele, performed a decision-making task where they made choices between receiving an amount of money for certain and taking a gamble. A strong bias was evident toward choosing the certain option when the option was phrased in terms of gains and toward gambling when the decision was phrased in terms of losses (the frame effect). Critically, this bias was significantly greater in the ss group compared with the lala group. In simultaneously acquired functional magnetic resonance imaging data, the ss group showed greater amygdala during choices made in accord, compared with those made counter to the frame, an effect not seen in the lala group. These differences were also mirrored by differences in anterior cingulateamygdala coupling between the genotype groups during decision making. Specifically, lala participants showed increased coupling during choices made counter to, relative to those made in accord with, the frame, with no such effect evident in ss participants. These data suggest that genetically mediated differences in prefrontal-amygdala interactions underpin interindividual differences in economic decision making.

\section{Introduction}

Human decision making can be biased by context and risk (Tversky and Kahneman, 1981; McNeil et al., 1982). For example, a supermarket might advertise yogurt as " $99 \%$ fat-free" instead of " $1 \%$ fat" to encourage sales, although these statements mean the same thing. This framing effect (Kahneman and Tversky, 1979) is conceptually embraced by prospect theory (Kahneman and Tversky, 2000). It is well recognized that individuals vary substantially in their susceptibility to decision-making biases, although the basis of this variability is unknown. The fact that these biases are observed across different cultures (Kahneman and Tversky, 1979; Sharp and Salter, 1997) and are difficult to eradicate even with training (McNeil et al., 1982) implies that hard-wired genetic

\footnotetext{
Received Jan. 26, 2009; revised Feb. 24, 2009; accepted Feb. $28,2009$.

This work was supported by a Wellcome Trust Programme Grant to R.J.D. B.d.M. was supported by a Wellcome Trust PhD studentship. We thank Ulrich Muller, Barbara Sahakian, and Trevor Robbins for assistance in recruiting participants, David Rubinsztein and Oana Sadiq for genotyping, and the Wellcome Trust Centre for Neuroimaging support staff for their help with data collection. J.P.R. was supported by the Raymond Way Fund. G.C.Y.T. was supported by the Agency for Science, Technology, and Research.

Correspondence should be addressed to either of the following: Raymond J. Dolan, Wellcome Trust Centre for Neuroimaging, 12 Queen Square, London WC1N 3BG, UK, E-mail: r.dolan@fil.ion.ucl.ac.uk; or Jonathan P. Roiser, Institute of Cognitive Neuroscience, University College London, 17 Queen Square, London WC1N 3AR, UK, E-mail: j.roiser@ucl.ac.uk.

DOI:10.1523/JNEUROSCI.0407-09.2009

Copyright $\odot 2009$ Society for Neuroscience $\quad 0270-6474 / 09 / 295985-07 \$ 15.00 / 0$
}

influences may play an important role in determining susceptibility to biases during decision making.

Previously, we demonstrated with functional magnetic resonance imaging (fMRI) that economic decisions influenced by framing were associated with responses in the amygdala, a key component of the brain's emotional system (De Martino et al., 2006; Dolan, 2007). The involvement of the amygdala is consistent with the hypothesis that such biases reflect the influence of basic emotional responses, or "affect heuristics," to guide behavior (Slovic et al., 2002). We reasoned that if the framing effect is indeed driven by amygdala activation then individuals exhibiting greater amygdala reactivity should be more susceptible to this decision-making bias.

Recent studies suggest that amygdala reactivity is strongly affected by genetic variation in the promoter region of the serotonin transporter gene (5-HTTLPR) (Lesch et al., 1996). Carriers of the short (s) allele at this locus, which results in reduced transcriptional efficacy and protein expression (Lesch et al., 1996), exhibit both increased resting-state amygdala blood flow (Canli et al., 2006) and greater amygdala reactivity to emotional stimuli (Hariri et al., 2002; Bertolino et al., 2005; Heinz et al., 2005; Munafò et al., 2008), relative to carriers homozygous for the long (1) allele. This variant also influences structure and function in the prefrontal cortex (PFC), particularly in subregions of the anterior cingulate cortex (ACC) (Heinz et al., 2005; Pezawas et al., 2005; Passamonti et al., 2008). These same regions are associated 
with resistance to the framing effect (De Martino et al., 2006), a finding consistent with their role in amygdala regulation (Phillips et al., 2003). Although some studies have reported effects of the 5-HTTLPR on decision making (Roiser et al., 2006; Blair et al., 2008; Homberg et al., 2008), the neural mechanisms responsible for differences in decision-making behavior between individuals of different 5-HTTLPR genotypes remain unclear.

Here, we tested the hypotheses that (1) variation at the 5-HTTLPR accounts for individual variability in the framing effect; (2) this effect is driven by elevated amygdala activity in s allele carriers; (3) this greater amygdala activity in s allele carriers results from inefficient regulation by the PFC.

\section{Materials and Methods}

Participants. Thirty right-handed participants of European ancestry were selected according to genotype at the 5-HTTLPR (Lesch et al., 1996). To maximize differences between the genotype groups, and improve statistical power, we included only participants homozygous for either the la allele or the s allele at the 5-HTTLPR; we excluded carriers of the rare lg allele, since this allele is thought to result in altered transcription relative to the la allele (Hu et al., 2006). All participants were screened for past psychiatric disorders using the Mini International Neuropsychiatric Inventory (Sheehan et al., 1998). Participants completed the Beck Depression Inventory (Beck et al., 1961), Impulsiveness, Venturesomeness, and Empathy questionnaire (Eysenck and Eysenck, 1991), State-Trait Anxiety Inventory (Spielberger et al., 1970), Need for Cognition Scale (Cacioppo et al., 1984), and Asperger's Questionnaire (Baron-Cohen et al., 2001), and intelligence quotient was estimated using the Wechsler Test of Adult Reading (Wechsler, 2001). The study was approved by the $\mathrm{Na}-$ tional Hospital for Neurology and Neurosurgery and the Institute of Neurology Joint Research Ethics Committee.

Experimental paradigm. The experiment was divided into three parts: an instruction, a scanning, and a postscan debriefing phase. In the instruction phase, the participants were familiarized with the decisionmaking task and given a number of practice trials. They were also told that during the task they would not receive feedback concerning the outcomes of their decisions but instead would receive a sum proportional to their total winnings at the end of the experiment (between $\mathfrak{E} 15$ and $\mathfrak{E} 60$ ). Hence, participants did not receive feedback on each trial.

The scanning phase of the experiment was divided into three sessions of 17 min each. Each session comprised 96 trials (32 loss frame, 32 gain frame, and 32 catch trials), ordered pseudorandomly, with a fixed intertrial interval of $10.7 \mathrm{~s}$. At the beginning of each trial, participants were shown a fixation cross $(0.7 \mathrm{~s})$, followed by a message indicating the initial amount of money which they received to play that trial (e.g., "You receive $\mathfrak{E 5 0 " ) ~ ( 2 ~ s ) . ~ F o u r ~ d i f f e r e n t ~ s t a r t i n g ~ a m o u n t s ~ w e r e ~ u s e d ~ i n ~ t h e ~ e x p e r i m e n t : ~}$ $\mathfrak{E} 25, \mathfrak{E} 50, \mathfrak{£} 75$, and $\mathfrak{1} 100$. Participants were instructed that they would not be able to retain the whole of this initial amount but would next have to choose between a sure and a gamble option $(4 \mathrm{~s})$. A blank screen was presented after the end of each trial ( $4 \mathrm{~s})$. The sure option was presented in gain frame trials as the amount of money retained from the starting

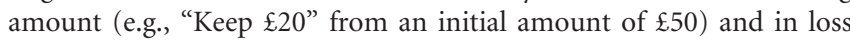
frame trials as the total amount of money lost from the starting amount

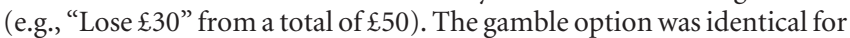
both frames and was represented by a pie chart depicting the probability of winning and losing in green and red, respectively (Fig. 1a). Four different probabilities were used in the experiment: 20, 40, 60, and $80 \%$. All experimental variables (total starting amount, percentage of the money offered, number of trials per session) were fully counterbalanced be- tween frame conditions. The expected value of the options were balanced in each trial (with the exception of the catch trials, see below) and mathematically equivalent between frames. For example, if participants initially received $\mathfrak{5} 50$, they were then required to choose between the op-

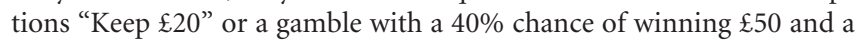
$60 \%$ chance of winning nothing. Participants were required to respond within $4 \mathrm{~s}$, using an MRI-compatible keypad, operated with their right hand.

Given the equivalence of the choices in terms of expected outcomes, we included "catch" trials ( 32 trials each session) to ensure that subjects remained actively engaged in the decision-making task throughout the course of the experiment. In these catch trials, in both frames, expected outcomes for the sure and gamble option were markedly unbalanced: in half of the trials ("gamble weighted"), the gamble option was preferable (e.g., $95 \%$ probability of winning by taking the gamble option versus a sure choice of $50 \%$ percent of the initial amount), and for the other half of trials ("sure weighted"), the sure option was preferable (e.g., $5 \%$ probability of winning by taking the gamble option versus a sure choice of $50 \%$ percent of the initial amount). As in the main experimental trials, the catch trials were also presented in either a gain or a loss frame.

At the end of the scanning session, the subjects were debriefed and asked about their strategies when performing the task and about their awareness of the frame manipulation. No participant reported a difference in difficulty between the two frames.

Analysis of demographic, personality, and demographic data. Demographic, personality, and behavioral data were analyzed using the Statistical Package for the Social Sciences 16 (SPSS). For demographic and personality measures, the genotype groups were compared using independent sample $t$ tests. Choice (indexed as proportion choices of the gamble option) and reaction time data from the decision-making task were analyzed using repeated-measures ANOVA with frame (gain/lose)

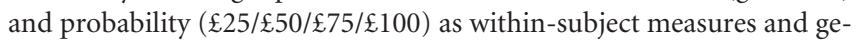
notype group (lala/ss) as the between-subjects measure. Choice and reaction time data from the catch trials were analyzed using repeatedmeasures ANOVA with frame (gain/lose) and weighting (keep/gamble) as within-subjects factors and genotype group (lala/ss) as the between subjects measure.

Image acquisition and analysis. Gradient-echo T2*-weighted images (echoplanar imaging) were acquired on a 3 Tesla head scanner (Magnetom Allegra; Siemens Medical), with 32 slices per volume using a $30^{\circ}$ titled acquisition sequence designed to reduce signal dropout in the orbitofrontal cortex and amygdala (Weiskopf et al., 2006). We positioned the slices to maximize coverage of the prefrontal cortex and subcortical structures, since these contained our primary regions of interest. Scanning parameters were as follows: echo time, $30 \mathrm{~ms}$; repetition time per slice, $65 \mathrm{~ms}$ (per volume $2080 \mathrm{~ms}$ ); slice thickness, $2 \mathrm{~mm}$; interslice gap, 1 
$\mathrm{mm}$; in-plane resolution, $2 \times 2 \mathrm{~mm}^{2}$. We collected 505 volumes per session per subject across three sessions.

Analysis was performed using Statistical Parametric Mapping (SPM5) in Matlab 7.1 (Wellcome Department of Imaging Neuroscience, London; www.fil.ion.ucl.ac.uk/spm). After discarding the first six image volumes from each session to allow for $\mathrm{T} 1$ equilibration effects, image volumes were realigned to the seventh image in the session, spatially normalized to the Montreal Neurological Institute (MNI) template and smoothed with a Gaussian kernel at $4 \mathrm{~mm}$ full-width at half-maximum (FWHM). Images were analyzed using an event-related design.

Regressors for each condition $\left(G_{\text {sure }}, G_{\text {gamble }}, L_{\text {sure }}\right.$ and $\left.L_{\text {gamble }}\right)$ were constructed on the basis of the participant's decisions in each frame and convolved with a synthetic hemodynamic response function, including time and dispersion derivatives, with onsets modeled as stick functions at the presentation of the decision. Low-frequency fluctuations were removed using a high-pass filter with a cutoff at $128 \mathrm{~s}$; correction for temporal autocorrelation was performed with an AR1 function, and global confounds were removed using global normalization. Parameter estimates for these four regressors were linearly combined to calculate the contrasts of interest in subject level analyses (see below). These contrast images were smoothed using a Gaussian kernel at $8 \mathrm{~mm}$ FWHM to account for individual anatomical variability and were used to compare the lala and ss genotype groups in random-effects analyses at the group level. T1-weighted structural images were coregistered with the realigned functional images, spatially normalized to the MNI template, and averaged across subjects to allow group-level anatomical localization.

The primary aim of our neuroimaging analysis was to identify brain regions exhibiting a genotype $\times$ frame $\times$ decision interaction, that is regions in which the genotype groups differed when we compared trials on which participants' choices followed the framing effect (risk aversion in the gain frame, $G_{\text {sure }}$; risk-seeking in the loss frame, $L_{\text {gamble }}$ ), to those on which participants' choices ran counter to the framing effect (risk seeking in the gain frame, $G_{\text {gamble }}$; risk aversion in the loss frame, $L_{\text {sure }}$ ) [framing effect contrast, $\left.\left(G_{\text {sure }}+L_{\text {gamble }}\right)-\left(L_{\text {sure }}+G_{\text {gamble }}\right)\right]$. We also sought to identify regions showing the opposite interaction [reverse framing effect contrast, $\left.\left(G_{\text {gamble }}+L_{\text {sure }}\right)-\left(L_{\text {gamble }}+G_{\text {sure }}\right)\right]$. These two-way interaction maps were computed for each participant at the subject level, and interactions with genotype group were calculated using independent samples $t$ tests at the group level.

To identify regions in which activity in the framing effect contrast correlated linearly with interindividual variation in susceptibility to the frame effect, we included each participant's susceptibility to the frame effect as a covariate in the group-level analyses. This value was calculated for each participant by subtracting the proportion of trials on which they chose the gamble option in the loss frame from the proportion of trials on which they chose the gamble option gain frame and linearly transforming the outcome to a value between 0 (most susceptible) and 1 (least susceptible).

The resulting $Z$-statistic images were thresholded at $Z>3.1$ corresponding to $p<0.001$ uncorrected. We discuss in the text results in a priori specified regions of interest (ROIs) [amygdala, anterior cingulate (Brodmann areas 24 and 32), and orbitofrontal cortex (Brodmann area 11)], which were based on our previous study (De Martino et al., 2006). To calculate small volume-adjusted family-wise error corrected (SVC) $p$ values across the ROIs above, we used the Wake Forest University Pickatlas toolbox (Maldjian et al., 2003). Activations in other regions are discussed in the text only if they survived whole-brain correction for multiple comparisons at $p<0.05$, but for completeness, all clusters where the peak voxel was significant at $Z>3.1$ outside the regions of interest are reported in supplemental Table S3, available at www.jneurosci.org as supplemental material. SPM images are displayed with $Z>2.6$ corresponding to $p<0.005$ (uncorrected).

Where we identified a significant three-way interaction, parameter estimates were extracted from the smoothed contrast images to identify significant simple main and interaction effects in a post hoc analysis. For all post hoc analyses, the $M S_{\text {error }}$ term for the three-way interaction effect and the $M S_{\text {model }}$ term for the effect of interest were calculated using repeated-measures ANOVA in SPSS 16, with frame (gain/lose) and decision (keep/gamble) as within-subject measures and genotype group (lala/ss) as the between-subjects measure. We then took the square root of the ratio of the $M S_{\text {model }}$ and $M S_{\text {error }}$ terms to produce a $t$ statistic and calculated $p$ values using the Student's $t$ cumulative distribution function in Matlab 7.1, based on the degrees of freedom for the error term from the three-way interaction effect.

We performed a psycho-physiological interaction (PPI) analysis by extracting the time series for the peak voxel identified in the genotype $X$ frame $\times$ decision interaction in the amygdala (supplemental Table S3, available at www.jneurosci.org as supplemental material) (physiological effect), extracting the regressors for the $G_{\text {gamble }}, G_{\text {sure }}, L_{\text {gamble }}$ and $L_{\text {sure }}$ trials, weighted $\left[\begin{array}{llll}-1 & 1 & 1 & -1\end{array}\right]$ (psychological effect), and taking the product of these two vectors (psychophysiological interaction) in SPM5 (Friston et al., 1997). PPI statistical parametric maps were created for each participant at the subject level and then combined at the group level to identify areas in which functional connectivity with the amygdala modulated by decision making differed between the genotype groups. The resulting $Z$-statistic images were thresholded at $Z>2.6$ corresponding to $p<0.005$ uncorrected. We discuss in the text results in a priori specified regions of interest (supragenual and subgenual anterior cingulate cortex), which were based on the study of Pezawas et al. (2005).

Genotyping. We used the protocol of Furlong et al. (1998) to genotype the 5-HTTLPR and identify participants homozygous for either the la or the s allele to participate in the behavioral experiment. We refrained from recruiting participants carrying the rare $\lg$ and sg allele, rs25531, since it appears to modify gene expression ( $\mathrm{Hu}$ et al., 2006), and we did not expect to have enough carriers to power a comparison of its effect. We, therefore, performed restriction enzyme digests with HpaII (Wendland et al., 2006) to identify and exclude lg or sg carriers.

PCR was performed across the 5-HTT promoter insertion/deletion using primers stpr5: $5^{\prime}$ ggc gtt gec gct ctg aat gc $3^{\prime}$ and stpr3: 5' gag gga ctg agc tgg aca acc ac 3' (Heils et al., 1996) (Invitrogen), with initial denaturation for $5 \mathrm{~min}$, followed by 35 cycles of $95^{\circ} \mathrm{C}$ for $0.5 \mathrm{~min}, 61^{\circ} \mathrm{C}$ for 0.5 $\min , 72^{\circ} \mathrm{C}$ for $0.5 \mathrm{~min}$, and final extension at $72^{\circ} \mathrm{C}$ for $10 \mathrm{~min}$. The $25 \mu \mathrm{l}$ reaction comprised $1 \mu \mathrm{lDNA}, 250 \mu \mathrm{M}$ dNTPs, $1 \mu \mathrm{l}$ enhancer (Molzym), $1 \times$ PCR buffer (Molzym), and 0.625 units Taq polymerase (Molzym). PCR product $(10 \mu \mathrm{l})$ was loaded on $1 \%$ UltraPure agarose gel stained with ethidium bromide and run for $1 \mathrm{~h}$ at $80 \mathrm{~V}$ in Tris-borate-EDTA buffer (TBE) with a $100 \mathrm{bp}$ ladder (Invitrogen) typing the $528 \mathrm{bp}$ and 484 bp products as the 1 and s alleles, respectively. PCR product $(10 \mu \mathrm{l})$ was digested by HpaII ( $5 \mathrm{U}$; New England Biolabs) in a $20 \mu \mathrm{l}$ reaction assay containing $1 \times$ NEBuffer 1 and $1 \times$ bovine serum albumin at $37^{\circ} \mathrm{C}$ for $3 \mathrm{~h}$. Restriction enzyme assay solution $(18 \mu \mathrm{l})$ were loaded on $4 \%$ UltraPure agarose gel and run for $2 \mathrm{~h}$ at $120 \mathrm{~V}$ in TBE with a $25 \mathrm{bp}$ ladder (Invitrogen), producing 298,126 , and $62 \mathrm{bp}$ fragments for the sa allele; 167,131 , 126 , and $62 \mathrm{bp}$ fragments for the sg allele; 341, 126, and $62 \mathrm{bp}$ fragments for the la allele; and 174, 167, 126, and $62 \mathrm{bp}$ fragments for the lg allele. The presence of a band at $167 \mathrm{bp}$ was thus used to detect and exclude lg and sg carriers from recruitment. For simplicity, we refer to the sa allele as the s allele since the sg allele is very rare (not detected in our sample).

\section{Results}

The participants were well matched in terms of demographic and personality measures (supplemental Table S1, available at www. jneurosci.org as supplemental material)

\section{Decision-making behavior}

Our decision-making paradigm featured mathematically identical decisions framed either in the context of winning (gain frame) or losing (lose frame) on different trials (Fig. 1a). Participants were biased toward risk aversion in the gain frame and risk seeking in the loss frame. On average, participants chose the sure option more often in the gain frame, gambling on $43.3 \pm 17.9 \%$ of trials, and the gamble option more often in the loss frame, gambling on $56.7 \pm 18.4 \%$ of trials. This "frame effect," i.e., the difference in risk-taking behavior between the loss and gain frames, was highly significant $\left(t_{(29)}=7.9, p<0.0000001\right)$, in keeping with predictions from prospect theory (Kahneman and 

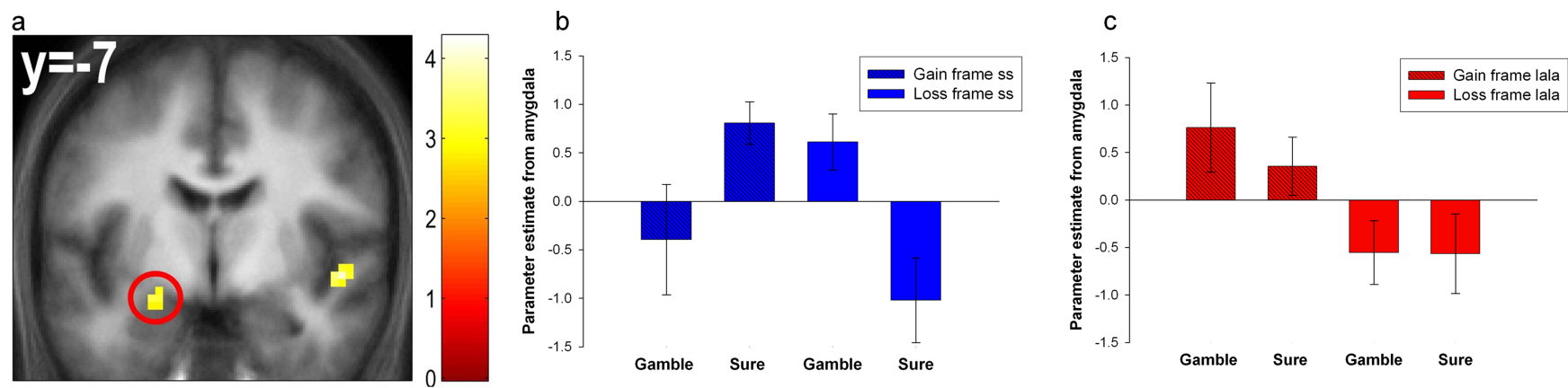

Figure 2. Difference in amygdala response evoked by decisions made in accord with the frame effect between the genotype groups. $\boldsymbol{a}, \mathrm{A}$ significant difference was detected between the two genotype groups in the frame effect contrast in left amygdala [circled in red; Talairach coordinates of peak voxel: $(x=-24, y=-4, z=-15)$ ]. Right is on the right of the image, and the color bar represents $t$ values. The image is thresholded at $p<0.005$ (uncorrected). $\boldsymbol{b}, \boldsymbol{c}$, Parameter estimate plots for the peak voxel of the amygdala interaction in the ss (red bars) and lala (blue bars) genotype groups. Participants in the ss genotype group exhibited greater amygdala response during decisions made in accord with, relative to those counter to, the frame effect in both the gain (hatched bars) and the loss (solid bars) frames. Amygdala response did not differ between decisions made in accord with and counter to the frame effect in the lala participants. Error bars represent SEM.

Tversky, 1979) and was greater when more money was at stake (supplemental Fig. S1, available at www.jneurosci.org as supplemental material).

In line with our principal behavioral prediction, we found a dramatic genetic effect on decision making. Specifically, we observed that the frame effect was significantly greater in the ss genotype compared with the lala genotype group $\left(t_{(28)}=2.1, p=\right.$ 0.048) (Fig. 1a). Note that there was no difference in overall risk-seeking behavior, indexed by total choice of the gamble option $(t<1)$ (supplemental Table S2, available at www. jneurosci.org as supplemental material). Reaction times were unaffected by frame, decision, or genotype, and all interactions with genotype were nonsignificant $(p>0.1)$, suggesting that difficulty was well matched between the frames, and similar for the genotype groups (supplemental Table S2, available at www. jneurosci.org as supplemental material). Participants also performed optimally on catch trials, with no difference between the genotype groups in either the gain or loss frames $(p>0.5$ for both) (supplemental Table S2, available at www.jneurosci.org as supplemental material).

\section{Analysis of fMRI data}

In line with our central hypothesis, a significant genotype $\times$ frame $X$ decision interaction was identified in the left amygdala $\left[t_{(28)}=3.5, p=0.042\right.$ (SVC) (Fig. 2). Analysis of the parameter estimates for each regressor, at the peak voxel in this cluster, revealed that the ss group exhibited a significant frame $\times$ decision interaction $\left(t_{(28)}=4.3, p<0.0001\right)$ (Fig. $\left.2 b\right)$, whereas the lala group did not $\left(t_{(28)}=0.60, p=0.28\right)$ (Fig. $\left.2 c\right)$. Further analysis of simple effects revealed that amygdala activation was greater both when ss participants chose the sure option relative to the gamble option in the gain frame $\left(t_{(28)}=2.6, p<0.01\right)$ and when they chose the gamble option relative to the sure option in the loss frame $\left(t_{(28)}=3.5, p<0.0001\right)$. A conjunction analysis confirmed overlapping amygdala responses when making choices in accord with the frame effect in the gain and the loss frames in the ss group (supplemental Fig. S2, available at www. jneurosci.org as supplemental material). Including susceptibility to the frame effect as a covariate in the analysis revealed that less susceptible participants exhibited greater activity in the orbitofrontal cortex (OFC) during decisions made in accord with the frame effect $\left[t_{(29)}=4.3, p=0.028\right.$ (SVC) $]$ (supplemental Fig. S3, available at www.jneurosci.org as supplemental material), replicating our previous finding (De Martino et al., 2006).
The reverse interaction $\left[\left(L_{\text {sure }}+G_{\text {gamble }}\right)-\left(G_{\text {sure }}+L_{\text {gamble }}\right)\right]$ identified genotype $\times$ frame $\times$ decision interactions in supragenual $\left[t_{(28)}=3.5, p=0.082(\mathrm{SVC})\right]$ and pregenual ACC $\left[t_{(28)}=3.7, p=0.083\right.$ (SVC) $]$ (supplemental Table S3 and supplemental Fig. S4a, available at www.jneurosci.org as supplemental material). Post hoc analysis of the parameter estimates for these interactions revealed that the ss group exhibited increased activation during decisions made counter to, relative to decisions made in accord with, the frame effect (supplemental Fig. S $4 b$, available at www.jneurosci.org as supplemental material). In contrast, the lala group showed either no difference in activation between the two decision types, or greater activation during decisions made in accord with the frame effect (supplemental Fig. S4c, available at www.jneurosci.org as supplemental material).

\section{Connectivity analysis of fMRI data}

An important concept arising out of studies of fear and fear extinction is that of prefrontal modulatory control of amygdala activity. To examine whether there was segregation in PFCamygdala modulatory coupling during decision making as a function of genotype group, we used a PPI analysis (Friston et al., 1997). PPI analysis enables the identification of brain regions in which functional coupling with another brain region differs according to experimental context. In the current experiment, this analysis permitted identification of regions where functional connectivity with the amygdala varied according to participants' decisions, and whether this modulation of connectivity differed between genotypes. For this analysis, we took the peak coordinate identified in the amygdala from the genotype $\times$ frame $\times$ decision interaction as our seed voxel.

Our PPI revealed subregions of PFC, including subgenual, pregenual, and supragenual ACC, where decision-dependent coupling with the amygdala differed as a function of genotype group (Fig. $3 a$ ). Analysis of the parameter estimates, at the peak voxel in the supragenual ACC, revealed that the PPI was significant only in the lala genotype group $(p<0.0005)$, whereas in the ss genotype group, coupling with the amygdala was not significantly modulated as a function of participants' decisions ( $p=$ 0.55). Further post hoc analysis, restricted to the lala genotype group, revealed that coupling with the amygdala increased during decisions made counter to, relative to those made in accord with, the frame effect $(p<0.01)$ (Fig. $3 b)$. This increase in coupling in the lala genotype group was significant in the loss frame 

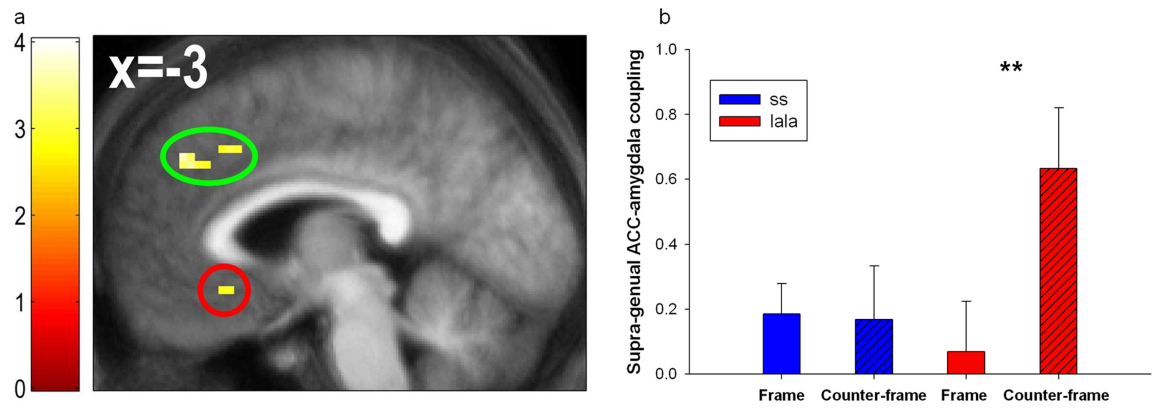

Figure 3. Differences between the genotype groups in amygdala-prefrontal coupling as a function of decision making (psychophysiological interaction). $\boldsymbol{a}$, In the lala participants, coupling between the amygdala and subgenual ACC [circled in red; Talairach coordinates of peak voxel: $(x=-3, y=26, z=-11)$ ], and amygdala and supragenual ACC [circled in green; Talairach coordinates of peak voxel: $(x=-3, y=43, z=34)$, was greater during decisions made counter to the frame effect relative to those made in accord with the frame effect. In the ss participants, there was either no difference in coupling between the two types of decisions or the opposite effect. Similar differences between the genotype groups were detected bilaterally in the OFC and pregenual ACC [not visible on this slice; Talairach coordinates of peak voxels: right OFC ( $x=36, y=37, z=-12)$; left OFC $(x=-30, y=30, z=-12)$; pregenual ACC $(x=6, y=45, z=3)]$. Right is on the right of the image, and the color bar represents $t$ values. The image is thresholded at $p<0.005$ (uncorrected). $\boldsymbol{b}$, Parameter estimate plots for the peak voxel of the supragenual ACC interaction. Supragenual ACC-amygdala coupling increased during decisions made counter to the frame effect in the lala genotype group but not in the ss genotype group. ${ }^{* *} p<0.01$. Error bars represent SEM.

( $L_{\text {sure }}$ vs $\left.L_{\text {gamble }} ; p<0.05\right)$ but not in the gain frame $\left(G_{\text {gamble }}\right.$ vs $G_{\text {sure }} ; p=0.39$ ).

In this PPI genotype interaction, the peak voxels localized to distinct regions of the ACC relative to the reverse frame effect interaction with genotype. To determine formally whether these loci overlapped, we performed a conjunction analysis between the PPI interaction with genotype and the reverse frame effect interaction with genotype. This analysis revealed that the regions identified in both interactions showed a topographical overlap in supragenual and pregenual ACC (supplemental Fig. S5, available at www.jneurosci.org as supplemental material). To examine this effect as a function of overall susceptibility to the frame effect, we asked whether this modulatory effect correlated with our index of susceptibility to the frame effect across subjects. A strong relationship was evident between decision-mediated pregenual ACC coupling with the amygdala and susceptibility to the frame effect $\left[t_{(29)}=4.75, p=0.009\right.$ (SVC) $]$ (supplemental Fig. S6 $a$, available at www.jneurosci.org as supplemental material); in simple terms, participants who did not show increased pregenual ACC-amygdala coupling during trials made counter to the frame effect were more susceptible (supplemental Fig. S6b, available at www. jneurosci.org as supplemental material).

\section{Discussion}

The key implication of our findings is that differences expressed at three distinct levels during economic decision making, namely behavior, brain activity, and PFC-amygdala functional coupling, segregate as a function of 5-HTTLPR genotype. Thus, the ss genotype group exhibited a greater frame effect, exhibited greater amygdala activity while making choices in accord with the frame effect, and failed to increase PFC-amygdala coupling while making choices counter to the frame effect. On this basis, we suggest that previously noted individual differences in decision making are underpinned by genetically mediated differences in amygdala reactivity and regulation.

Our finding of amygdala responses during choices in accord with the frame effect in both the gain and the loss frames in the ss participants supports the hypothesis that biases in both frames are likely to be related to a unitary process driven by automatic emotional responses to contextual cues (Kahneman and Freder- ick 2007). However, ACC responses in the reverse frame effect contrast were greater in the loss condition than the gain condition in the ss genotype group; furthermore, the PPI interaction with genotype appeared to be driven primarily by elevated ACCamygdala coupling during decisions made counter to the frame effect in the loss frame in the lala genotype group. These findings raise the possibility that different processes might underlie resistance to the frame effect in the gain and loss conditions. This effect warrants further investigation in future studies.

Previous studies have suggested that risk aversion may be mediated by responses in regions other than the amygdala, in particular the insula $(\mathrm{Ku}-$ hnen and Knutson, 2005; Liu et al., 2007), consistent with the suggestion that enhanced sensitivity to losses is driven by negative emotions (Camerer, 2005). In the present study, we could not identify insula responses when analyzing the frame effect either in the gain condition or the loss condition, even when we restricted the analysis to the ss genotype group (data not shown). However, our study differs from those aforementioned in that we did not provide feedback during the task. An apparent discrepancy in findings might be reconciled by a suggestion that insula responses are more associated with situations where there is an immediate, rather than a temporally distant, risk of aversive outcomes. We note a recent study reported that loss aversion was not associated with amygdala activity (Tom et al., 2007). This finding conflicts with our current study and previous work (Kahn et al., 2002; Kuhnen and Knutson, 2005; De Martino et al., 2006), which indicated the amygdala plays a central role in the neural computation of potential losses and may be involved in generating the phenomenon of loss aversion.

Although the effect of 5-HTTLPR genotype on serotonin transporter protein expression and extracellular serotonin concentration in humans remains unclear (Shioe et al., 2003), it appears carriers of the s allele exhibit differences relative to 11 homozygotes in terms of personality, behavior, and brain structure and function (Caspi et al., 2003; Sen et al., 2004; Pezawas et al., 2005; Canli et al., 2006). This genotype may not directly affect extra-cellular serotonin concentration in adults but instead may influence cortical development through regulation of synaptic plasticity (Gaspar et al., 2003; Pezawas et al., 2005). Consistent with this hypothesis, recent studies suggested that carriers of the $\mathrm{s}$ allele have decreased gray matter volume in the ACC along with decreased amygdala-ACC functional connectivity (Pezawas et al., 2005) and increased amygdala responsitivity to fearful faces (Hariri et al., 2005; Munafò et al., 2008).

The ACC plays an important regulatory role in processing of emotional information, possibly reflecting strong inhibitory connections between this region and the amygdala (Phillips et al., 2003; Rosenkranz et al., 2003). A previous study reported reduced functional connectivity between the ACC and amygdala in carriers of the s allele, independent of task demands (Pezawas et al., 2005). Our PPI analysis allowed us to extend this finding to the context of behavioral variability in decision making. The lala 
group exhibited significantly greater coupling between a number of ACC regions and the amygdala during decisions made counter to the frame effect, whereas coupling between these structures did not differ according to decision-making behavior in the ss group. Notably, coupling with the amygdala and pregenual ACC was also strongly related to susceptibility to the frame effect between subjects, independent of genotype.

Our data suggest that regions of the ACC implicated in cognitive control regulate amygdala activity, providing a mechanism by which analytic decision-making processes might override basic emotional responses, enabling resistance to the frame effect. This is in keeping with evidence that the ACC receives inputs from both the amygdala and OFC, which tracks the value of stimuli and choices (Schoenbaum et al., 2006), to integrate emotional and analytic information to guide goal-directed behavior. We speculate that the lala participants were able to dynamically regulate amygdala activity more efficiently than the ss participants. At the neuronal level, these effects might reflect a variety of differences between the genotype groups, including altered white matter connections between the ACC and amygdala, or altered synaptic plasticity at the amygdala level involving inputs from the ACC. However, two important caveats to this interpretation merit comment: (1) a PPI analysis does not permit an assignment of directionality; (2) although the ACC regions identified in the categorical fMRI and PPI genotype interactions overlapped to a small extent, the peak voxels were somewhat distant, suggesting that the effects identified in these regions may be driven by different neuronal assemblies (Beckmann et al., 2009). The precise role played by different ACC regions in regulating amygdala activity clearly warrants further investigation.

The finding of enhanced amygdala responses only in the ss group might seem surprising in light of the finding of an overall group effect in the study of De Martino et al. (2006). Note that in the current study, we only included individuals homozygous for either the s or the la allele at the 5-HTTLPR; heterozygotes, who make up at least half of the population, were excluded. Therefore, $\sim 75 \%$ of the subjects tested by De Martino et al. (2006) would be expected to carry at least one copy of the s or functionally equivalent $\lg$ allele, whereas only $\sim 25 \%$ would be expected to be homozygous for la allele (i.e., 5 of 20 subjects). A previous study suggested that heterozygous individuals performed intermediate between 11 and ss carriers on a similar decision-making task (Roiser et al., 2006). Therefore, we would expect individuals carrying the s or functionally equivalent lg allele (Hu et al., 2006), as well as the la allele, to show slightly smaller, but nevertheless significant, amygdala responses relative to ss carriers while making choices in accord with the frame effect. However, this prediction requires testing in future studies.

Several factors may contribute to the frame effect (e.g., outcome salience, probability distortion, the representation of risk prospect) (for a detailed review, see Kühberger, 1998). However, since our investigation was motivated by the finding of De Martino et al. (2006), and used the same task, our primary hypothesis was that such a bias (in the specific context of our task) is likely to reflect the influence of basic emotional responses to guide behavior (Slovic et al., 2002), as recently proposed by Kahneman and Frederick (2007). Nevertheless, our results do not rule out the possibility that nonemotional processes may contribute to the behavioral effect we found. Indeed, such processes may have driven the small framing effect we observed in the lala group, which was not associated with amygdala responses.

It has long been known that individuals differ in terms of their susceptibility to contextually mediated biases in decision making, although the basis of such variability has remained obscure. Our data suggest that a key source of variation is genetic, in particular that individuals of the ss genotype are more susceptible to biases driven by automatic amygdala responses to contextual stimuli and that one mechanism underlying this effect may be reduced prefrontal regulation of amygdala activity.

\section{References}

Baron-Cohen S, Wheelwright S, Skinner R, Martin J, Clubley E (2001) The autism-spectrum quotient (AQ): evidence from Asperger syndrome/ high-functioning autism, males and females, scientists and mathematicians. J Autism Dev Disord 31:5-17.

Beck AT, Ward CH, Medelson M, Erbaugh J (1961) An inventory for measuring depression. Arch Gen Psychiatry 4:561-571.

Beckmann M, Johansen-Berg H, Rushworth MF (2009) Connectivity-based parcellation of human cingulate cortex and its relation to functional specialization. J Neurosci 29:1175-1190.

Bertolino A, Arciero G, Rubino V, Latorre V, De Candia M, Mazzola V, Blasi G, Caforio G, Hariri A, Kolachana B, Nardini M, Weinberger DR, Scarabino T (2005) Variation of human amygdala response during threatening stimuli as a function of 5'HTTLPR genotype and personality style. Biol Psychiatry 57:1517-1525.

Blair KS, Finger E, Marsh AA, Morton J, Mondillo K, Buzas B, Goldman D, Drevets WC, Blair RJ (2008) The role of 5-HTTLPR in choosing the lesser of two evils, the better of two goods: examining the impact of 5-HTTLPR genotype and tryptophan depletion in object choice. Psychopharmacology (Berl) 196:29-38.

Cacioppo JT, Petty RE, Kao CF (1984) The efficient assessment of need for cognition. J Pers Assess 48:306-307.

Camerer CF (2005) Three cheers - psychological, theoretical, empirical - for loss aversion. J Mark Res 42:129-133.

Canli T, Qiu M, Omura K, Congdon E, Haas BW, Amin Z, Herrmann MJ, Constable RT, Lesch KP (2006) Neural correlates of epigenesis. Proc Natl Acad Sci U S A 103:16033-16038.

Caspi A, Sugden K, Moffitt TE, Taylor A, Craig IW, Harrington H, McClay J, Mill J, Martin J, Braithwaite A, Poulton R (2003) Influence of life stress on depression: moderation by a polymorphism in the 5-HTT gene. Science 301:386-389.

De Martino B, Kumaran D, Seymour B, Dolan RJ (2006) Frames, biases, and rational decision-making in the human brain. Science 313:684-687.

Dolan RJ (2007) The human amygdala and orbital prefrontal cortex in behavioural regulation. Philos Trans R Soc Lond B Biol Sci 362:787-799.

Eysenck HJ, Eysenck SBG (1991) Adult impulsiveness, venturesomeness and empathy scale. London: Hodder Soughton.

Friston KJ, Buechel C, Fink GR, Morris J, Rolls E, Dolan RJ (1997) Psychophysiological and modulatory interactions in neuroimaging. Neuroimage 6:218-229.

Furlong RA, Ho L, Walsh C, Rubinsztein JS, Jain S, Paykel ES, Easton DF, Rubinsztein DC (1998) Analysis and meta-analysis of two serotonin transporter gene polymorphisms in bipolar and unipolar affective disorders. Am J Med Genet 81:58-63.

Gaspar P, Cases O, Maroteaux L (2003) The developmental role of serotonin: news from mouse molecular genetics. Nat Rev Neurosci 4:1002-1012.

Hariri AR, Mattay VS, Tessitore A, Kolachana B, Fera F, Goldman D, Egan MF, Weinberger DR (2002) Serotonin transporter genetic variation and the response of the human amygdala. Science 297:400-403.

Hariri AR, Drabant EM, Munoz KE, Kolachana BS, Mattay VS, Egan MF, Weinberger DR (2005) A susceptibility gene for affective disorders and the response of the human amygdala. Arch Gen Psychiatry 62:146-152.

Heils A, Teufel A, Petri S, Stober G, Riederer P, Bengel D, Lesch KP (1996) Allelic variation of human serotonin transporter gene expression. J Neurochem 66:2621-2624.

Heinz A, Braus DF, Smolka MN, Wrase J, Puls I, Hermann D, Klein S, Grüsser SM, Flor H, Schumann G, Mann K, Büchel C (2005) Amygdalaprefrontal coupling depends on a genetic variation of the serotonin transporter. Nat Neurosci 8:20-21.

Homberg JR, van den Bos R, den Heijer E, Suer R, Cuppen E (2008) Serotonin transporter dosage modulates long-term decision-making in rat and human. Neuropharmacology 55:80-84.

Hu XZ, Lipsky RH, Zhu G, Akhtar LA, Taubman J, Greenberg BD, Xu K, Arnold PD, Richter MA, Kennedy JL, Murphy DL, Goldman D (2006) 
Serotonin transporter promoter gain-of-function genotypes are linked to obsessive-compulsive disorder. Am J Hum Genet 78:815-826.

Kahn I, Yeshurun Y, Rotshtein P, Fried I, Ben-Bashat D, Hendler T (2002) The role of the amygdala in signaling prospective outcome of choice. Neuron 33:983-994.

Kahneman D, Frederick S (2007) Frames and brains: elicitation and control of response tendencies. Trends Cogn Sci 11:45-46.

Kahneman D, Tversky A (1979) Prospect: an analysis of decision-making. Econometria 47:263-291.

Kahneman D, Tversky A (2000) Choices, values and frames. New York: Cambridge UP.

Kühberger A (1998) The influence of framing on risky decisions: a metaanalysis. Organ Behav Hum Decis Process 75:23-55.

Kuhnen CM, Knutson B (2005) The neural basis of financial risk taking. Neuron 47:763-770.

Lesch KP, Bengel D, Heils A, Sabol SZ, Greenberg BD, Petri S, Benjamin J, Müller CR, Hamer DH, Murphy DL (1996) Association of anxietyrelated traits with a polymorphism in the serotonin transporter gene regulatory region. Science 274:1527-1531.

Liu X, Powell DK, Wang H, Gold BT, Corbly CR, Joseph JE (2007) Functional dissociation in frontal and striatal areas for processing of positive and negative reward information. J Neurosci 27:4587-4597.

Maldjian JA, Laurienti PJ, Kraft RA, Burdette JH (2003) An automated method for neuroanatomic and cytoarchitectonic atlas-based interrogation of fMRI data sets. Neuroimage 19:1233-1239.

McNeil BJ, Pauker SG, Sox HC Jr, Tversky A (1982) On the elicitation of preferences for alternative therapies. N Engl J Med 306:1259-1262.

Munafò MR, Brown SM, Hariri AR (2008) Serotonin transporter (5HTTLPR) genotype and amygdala activation: a meta-analysis. Biol Psychiatry 63:852-857.

Passamonti L, Cerasa A, Gioia MC, Magariello A, Muglia M, Quattrone A, Fera F (2008) Genetically dependent modulation of serotonergic inactivation in the human prefrontal cortex. Neuroimage 40:1264-1273.

Pezawas L, Meyer-Lindenberg A, Drabant EM, Verchinski BA, Munoz KE, Kolachana BS, Egan MF, Mattay VS, Hariri AR, Weinberger DR (2005) 5-HTTLPR polymorphism impacts human cingulate-amygdala interactions: a genetic susceptibility mechanism for depression. Nat Neurosci 8:828-834.

Phillips ML, Drevets WC, Rauch SL, Lane R (2003) Neurobiology of emotion perception. II: Implications for major psychiatric disorders. Biol Psychiatry 54:515-528.

Roiser JP, Rogers RD, Cook LJ, Sahakian BJ (2006) The effect of polymor- phism at the serotonin transporter gene on decision-making, memory and executive function in ecstasy users and controls. Psychopharmacology (Berl) 188:213-227.

Rosenkranz JA, Moore H, Grace AA (2003) The prefrontal cortex regulates lateral amygdala neuronal plasticity and responses to previously conditioned stimuli. J Neurosci 23:11054-11064.

Schoenbaum G, Roesch MR, Stalnaker TA (2006) Orbitofrontal cortex, decision-making and drug addiction. Trends Neurosci 29:116-124.

Sen S, Burmeister M, Ghosh D (2004) Meta-analysis of the association between a serotonin transporter promoter polymorphism (5-HTTLPR) and anxiety-related personality traits. Am J Med Genet B Neuropsychiatr Genet 127B:85-89.

Sharp DJ, Salter SB (1997) Project escalation and sunk costs: a test of the international generalizability of agency and prospect theories. J Inter Bus Stud 28:101-121.

Sheehan DV, Lecrubier Y, Sheehan KH, Amorim P, Janavs J, Weiller E, Hergueta T, Baker R, Dunbar GC (1998) The Mini-International Neuropsychiatric Interview (M.I.N.I.): the development and validation of a structured diagnostic psychiatric interview for DSM-IV and ICD-10. J Clin Psychiatry 59 [Suppl] 20:22-33;quiz 34-57.

Shioe K, Ichimiya T, Suhara T, Takano A, Sudo Y, Yasuno F, Hirano M, Shinohara M, Kagami M, Okubo Y, Nankai M, Kanba S (2003) No association between genotype of the promoter region of serotonin transporter gene and serotonin transporter binding in human brain measured by PET. Synapse 48:184-188.

Slovic P, Finucane E, Perers M, MacGregor D (2002) The affect heuristic. In: Heuristics and biases: the psychology of intuituve judgment (Gilovich T, Griffin DW, Kahneman D, eds), pp 397-421. New York: Cambridge UP.

Spielberger CD, Gorsuch RL, Lushene RE (1970) Manual for the state-trait anxiety inventory. Palo Alto, California: Consulting Psychologists.

Tom SM, Fox CR, Trepel C, Poldrack RA (2007) The neural basis of loss aversion in decision-making under risk. Science 315:515-518.

Tversky A, Kahneman D (1981) The framing of decisions and the psychology of choice. Science 211:453-458.

Wechsler D (2001) Wechsler test of adult reading manual. San Antonio, TX: Psychological Corporation.

Weiskopf N, Hutton C, Josephs O, Deichmann R (2006) Optimal EPI parameters for reduction of susceptibility-induced BOLD sensitivity losses: a whole-brain analysis at $3 \mathrm{~T}$ and $1.5 \mathrm{~T}$. Neuroimage 33:493-504.

Wendland JR, Martin BJ, Kruse MR, Lesch KP, Murphy DL (2006) Simultaneous genotyping of four functional loci of human SLC6A4, with a reappraisal of 5-HTTLPR and rs25531. Mol Psychiatry 11:224-226. 